\title{
Simulation of Mixing Process in Drum Mixer with Different Topology of Material Flows
}

\author{
D.M. Borodulin \\ Doctor of Technical Sciences, Head of the Department \\ technological design of food production \\ Kemerovo State University \\ Kemerovo, Russia Federation \\ borodulin_dmitri@list.ru \\ D.V. Sukhorukov \\ Candidate of Technical Sciences, Department \\ technological design of food production \\ Kemerovo State University \\ Kemerovo, Russia Federation \\ pioner_dias@mail.ru
}

\author{
I.A. Bakin \\ Doctor of Technical Sciences, Department \\ technological design of food production \\ Kemerovo State University \\ Kemerovo, Russia Federation \\ bakin_ia@mail.ru \\ S.A. Ratnikov \\ Candidate of Technical Sciences, Department \\ technological design of food production \\ Kemerovo State University \\ Kemerovo, Russia Federation \\ tanyrat@mail.ru
}

\begin{abstract}
The article contains a mathematical description of the process of mixing bulk materials in a drum aggregate using elements of mathematical statistics, theory of probability and averaging models. A random stationary process is a flow that is formed by means of dispensers in this case. The correlation function is used as its parameter. It is a measure of the stability of the process. The relationship between the variances of the input and output signals is established by means of a correlation analysis. It allows predicting the degree of homogeneity of the material flow (smoothing ability of the mixer) at the output for the given parameters of the input signal: the coefficients of recycling, the time of its presence in the mixer and the dispersion of the input signal. The correlation analysis of the flow of material allowed the development of a new design of a continuous drum mixer. As a result, we found that the organization of external and internal recycling circuits greatly affects the reduction of fluctuations in the input signal. This reduces the variance of components and increases homogeneity of the mixture.
\end{abstract}

Keywords - smoothing ability, variance, drum mixer, mixing, recycling, bulk material

\section{INTRODUCTION}

At present, the requirements for the quality of the product have risen sharply. This is due to the constantly growing level and pace of life of the population. Therefore, it is advisable to develop new designs of continuous mixers [2,11] equipped with suitable metering devices.

Drum mixers produce high-quality mixtures with a $1: 40$ component ratio, a vibrating ratio of $1: 100$, and centrifugal in the range of $1: 100$ to $1: 500$.

These devices are characterized by high smoothing ability, low specific metal and energy costs, high intensity of the mixing process, due to the organization of directed movement of thin-layer material flows in combination with the advance and recycling circuits. These devices are suitable as the main component for a continuous mixing plant, either individually or in any combination thereof [5,9]. If it is necessary to prepare mixtures, for example, with a ratio of ingredients of 1 : 1000 , it is necessary to consistently install continuously operating drum mixers (ratio of components 1:10) and vibration (at a ratio of $1: 100)$ types.

The use of correlation analysis is possible when simulating the process of mixing in vibration and centrifugal mixers [4, $6]$. We use a similar approach for a continuous drum mixer. The literary review made it possible to conclude that the input feeding material flows can be regarded as random stationary processes. On the basis of this, the mathematical expectation of the instantaneous rate of components in time can be considered constant with practice accuracy. In this case, the considered pulsations of feeding material flows have in addition to a random and periodic character.

All this allows us to use the theory of time series along with a cybernetic approach for constructing a mathematical model of a mixing aggregate. For the analysis of time series, it is proposed to use various methods, the most common of which are:

- correlation method;

- spectral method;

- method of smoothing and filtering;

- autoregressive models.

We chose a correlation analysis to mathematically simulate the process of mixing the bulk components within the allowed limits of the theory of time series. In this case, the correlation function is a parameter of a random stationary process. Correlation analysis establishes the relationship between the variances of the input and output signals, predicts the 
smoothing capacity of the mixer at the output with the given recycling factors.

\section{OBJECTS AND METHODS OF RESEARCH}

Analysis of two flow patterns of material flows, allowed one to develop a new original design of a continuous drum mixer [8]. The peculiarity of this design is that inside the mixer there is a shaft with $\Gamma$-shaped blades for increasing the smoothing ability as a result of internal recycling of the mixture.

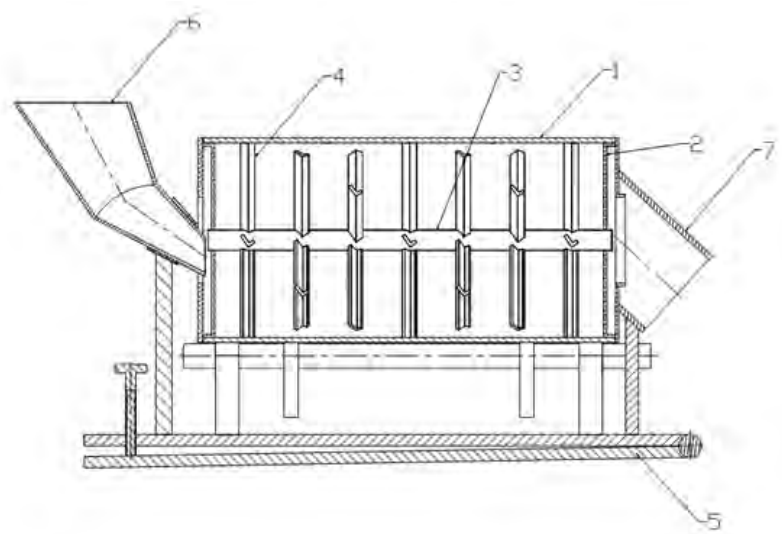

Fig. 1 Drum mixer: 1-drum; 2-centering supports; 3- central shaft; 4- Гshaped blades; 5-frame; 6-feed nozzle; 7-discharge nozzle

The drum mixer operates as follows $[3,8,9]$. Bulk materials are fed into the mixer through the feed nozzle 6 . As the drum 1 rotates, the powdery materials are poured from the working surfaces of the $\Gamma$-shaped blades downward, moving simultaneously along the two generatrixes of the drum 1 . In addition to dividing the volume of the material into two unequal flows on each blade, along the length of the mixing drum. The main flow of the mixture will move axially towards the discharge side [15]. Due to the possibility of turning the $\Gamma$ shaped blades relative to each other by $360^{\circ}$, they can be installed in staggered or spiral-like order $[10,12]$. As a result, multiple overlapping flows occur, favoring a general averaging of the quality of the mixture. The finished mixture is discharged through a discharge nozzle 7.

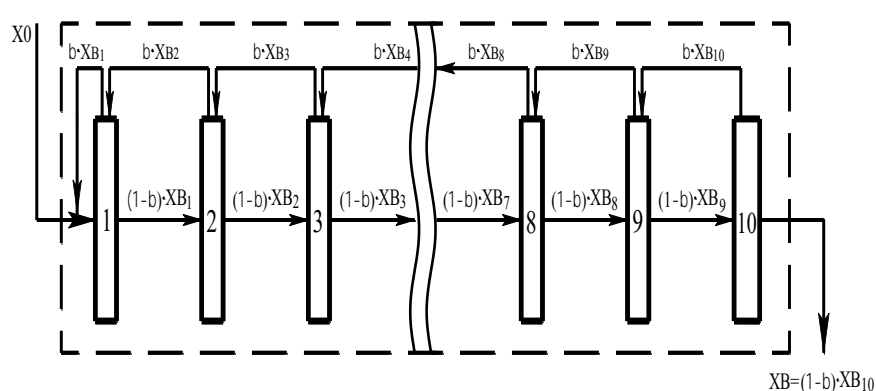

Fig. 2 A scheme of motion of material flows in the helical arrangement of $\Gamma$ shaped blades in a drum mixer

\section{RESULTS AND DISCUSSION}

First we will consider the first scheme of the flow of material flows with a helical arrangement of $\Gamma$-shaped blades inside the drum, shown in Fig. 2. It recirculates material flows with their subsequent superposition on the main flow on each of the ten blades corresponding to certain cells of the mixer. Material flows are described by random stationary ergodic functions, so we can write a system of material balance equations for this case:

$$
\left\{\begin{array}{l}
X_{1}=X 0+\beta \cdot X B_{1}, \\
X_{2}=(1-\beta) \cdot X B_{1}+\beta \cdot X B_{2}, \\
X_{3}=(1-\beta) \cdot X B_{2}+\beta \cdot X B_{3}, \\
X_{4}=(1-\beta) \cdot X B_{3}+\beta \cdot X B_{4}, \\
X_{5}=(1-\beta) \cdot X B_{4}+\beta \cdot X B_{5}, \\
X_{6}=(1-\beta) \cdot X B_{5}+\beta \cdot X B_{6}, \\
X_{7}=(1-\beta) \cdot X B_{6}+\beta \cdot X B_{7}, \\
X_{8}=(1-\beta) \cdot X B_{7}+\beta \cdot X B_{8}, \\
X_{9}=(1-\beta) \cdot X B_{8}+\beta \cdot X B_{9}, \\
X_{10}=(1-\beta) \cdot X B_{9}+\beta \cdot X B_{10}, \\
X B=(1-\beta) \cdot X B_{10}
\end{array}\right.
$$

where: $\mathrm{X} 0$ - flow of material entering the mixer;

$\mathrm{Xi}$ - flow of material entering the $\mathrm{i}$-stage of the mixer, $\mathrm{i}=1 \ldots \mathrm{n}$;

$\mathrm{XBi}$ - flow of material leaving the i-stage of the mixer,

$\mathrm{i}=1 \ldots \mathrm{n}$;

$\mathrm{XB}$ - flow of material leaving the mixer;

$\beta$ - coefficient of recycling;

$\mathrm{n}$ - number of stages;

$\tau$ - correlation interval.

On the basis of expression (1), we can write a system of equations that determine the correlation functions of the flows in the absence of their mutual correlation:

$$
\left\{\begin{array}{l}
\mathrm{K}_{\mathrm{X} 1}(\tau)=\mathrm{K}_{\mathrm{X} 0}(\tau)+\beta^{2} \cdot \mathrm{K}_{\mathrm{XB1}}(\tau), \\
\mathrm{K}_{\mathrm{X} 2}(\tau)=(1-\beta)^{2} \cdot \mathrm{K}_{\mathrm{XB1}}(\tau)+\beta^{2} \cdot \mathrm{K}_{\mathrm{XB} 2}(\tau), \\
\mathrm{K}_{\mathrm{X} 3}(\tau)=(1-\beta)^{2} \cdot \mathrm{K}_{\mathrm{XB} 2}(\tau)+\beta^{2} \cdot \mathrm{K}_{\mathrm{XB} 3}(\tau), \\
\mathrm{K}_{\mathrm{X} 4}(\tau)=(1-\beta)^{2} \cdot \mathrm{K}_{\mathrm{XB} 3}(\tau)+\beta^{2} \cdot \mathrm{K}_{\mathrm{XB} 4}(\tau), \\
\mathrm{K}_{\mathrm{X} 5}(\tau)=(1-\beta)^{2} \cdot \mathrm{K}_{\mathrm{XB} 4}(\tau)+\beta^{2} \cdot \mathrm{K}_{\mathrm{XB} 5}(\tau), \\
\mathrm{K}_{\mathrm{X} 6}(\tau)=(1-\beta)^{2} \cdot \mathrm{K}_{\mathrm{XB} 5}(\tau)+\beta^{2} \cdot \mathrm{K}_{\mathrm{XB} 6}(\tau), \\
\mathrm{K}_{\mathrm{X} 7}(\tau)=(1-\beta)^{2} \cdot \mathrm{K}_{\mathrm{XB} 6}(\tau)+\beta^{2} \cdot \mathrm{K}_{\mathrm{XB} 7}(\tau), \\
\mathrm{K}_{\mathrm{X} 8}(\tau)=(1-\beta)^{2} \cdot \mathrm{K}_{\mathrm{XB} 7}(\tau)+\beta^{2} \cdot \mathrm{K}_{\mathrm{XB8}}(\tau), \\
\mathrm{K}_{\mathrm{X} 9}(\tau)=(1-\beta)^{2} \cdot \mathrm{K}_{\mathrm{XB} 8}(\tau)+\beta^{2} \cdot \mathrm{K}_{\mathrm{XB} 9}(\tau), \\
\mathrm{K}_{\mathrm{X} 10}(\tau)=(1-\beta)^{2} \cdot \mathrm{K}_{\mathrm{XB} 9}(\tau)+\beta^{2} \cdot \mathrm{K}_{\mathrm{XB10}}(\tau), \\
\mathrm{K}_{\mathrm{XB}}(\tau)=(1-\beta)^{2} \cdot \mathrm{K}_{\mathrm{XB10}}(\tau)
\end{array}\right.
$$


where: $K X O(\tau)$ - correlation function of the flow of material entering the mixer;

$K X i(\tau)$ - correlation function of the flow of material entering the $\mathrm{i}$-stage of the mixer, $\mathrm{i}=1 \ldots \mathrm{n}$;

$\operatorname{KXBi}(\tau)$ - correlation function of the flow of material leaving the $\mathrm{i}$-stage of the mixer, $\mathrm{i}=1 \ldots \mathrm{n}$;

$K X B(\tau)$ - correlation function of the flow of material leaving the mixer.

The assumption is that $K_{X B i}(\tau)=K_{X i}(\tau)$. Therefore, there is no process of averaging the fluxes. Then system (2) can be written in the form:

$$
\left\{\begin{array}{l}
\mathrm{K}_{\mathrm{X} 1}(\tau)=\mathrm{K}_{\mathrm{X} 0}(\tau)+\beta^{2} \cdot \mathrm{K}_{\mathrm{X} 1}(\tau), \\
\mathrm{K}_{\mathrm{X} 2}(\tau)=(1-\beta)^{2} \cdot \mathrm{K}_{\mathrm{X} 1}(\tau)+\beta^{2} \cdot \mathrm{K}_{\mathrm{XB} 2}(\tau), \\
\mathrm{K}_{\mathrm{X} 3}(\tau)=(1-\beta)^{2} \cdot \mathrm{K}_{\mathrm{X} 2}(\tau)+\beta^{2} \cdot \mathrm{K}_{\mathrm{XB3}}(\tau), \\
\mathrm{K}_{\mathrm{X} 4}(\tau)=(1-\beta)^{2} \cdot \mathrm{K}_{\mathrm{X} 3}(\tau)+\beta^{2} \cdot \mathrm{K}_{\mathrm{XB} 4}(\tau), \\
\mathrm{K}_{\mathrm{X} 5}(\tau)=(1-\beta)^{2} \cdot \mathrm{K}_{\mathrm{X} 4}(\tau)+\beta^{2} \cdot \mathrm{K}_{\mathrm{XB5}}(\tau), \\
\mathrm{K}_{\mathrm{X} 6}(\tau)=(1-\beta)^{2} \cdot \mathrm{K}_{\mathrm{X} 5}(\tau)+\beta^{2} \cdot \mathrm{K}_{\mathrm{XB6}}(\tau), \\
\mathrm{K}_{\mathrm{X} 7}(\tau)=(1-\beta)^{2} \cdot \mathrm{K}_{\mathrm{X} 6}(\tau)+\beta^{2} \cdot \mathrm{K}_{\mathrm{XB} 7}(\tau), \\
\mathrm{K}_{\mathrm{X} 8}(\tau)=(1-\beta)^{2} \cdot \mathrm{K}_{\mathrm{X} 7}(\tau)+\beta^{2} \cdot \mathrm{K}_{\mathrm{XB8}}(\tau), \\
\mathrm{K}_{\mathrm{X} 9}(\tau)=(1-\beta)^{2} \cdot \mathrm{K}_{\mathrm{X} 8}(\tau)+\beta^{2} \cdot \mathrm{K}_{\mathrm{XB9}}(\tau), \\
\mathrm{K}_{\mathrm{X} 10}(\tau)=(1-\beta)^{2} \cdot \mathrm{K}_{\mathrm{X} 9}(\tau)+\beta^{2} \cdot \mathrm{K}_{\mathrm{XB10}}(\tau), \\
\mathrm{K}_{\mathrm{XB}}(\tau)=\mathrm{K}_{\mathrm{X} 10}(\tau)
\end{array}\right.
$$

Solving the system (3), we obtain:

$$
\begin{aligned}
& K_{X B}(\tau)=K_{X 0}(\tau) \cdot \frac{1-\beta}{1+\beta} \cdot \frac{1-\beta}{1+\beta} \cdot \frac{1-\beta}{1+\beta} \cdot \frac{1-\beta}{1+\beta} \cdot \frac{1-\beta}{1+\beta} \cdot \frac{1-\beta}{1+\beta} \times \\
& \times \frac{1-\beta}{1+\beta} \cdot \frac{1-\beta}{1+\beta} \cdot \frac{1-\beta}{1+\beta} \frac{1-\beta}{1+\beta}
\end{aligned}
$$

or:

$$
K_{X B}(\tau)=K_{X 0}(\tau) \cdot\left(\frac{1-\beta}{1+\beta}\right)^{10}
$$

It is known that the $\tau=0$ is equal to the correlation function of a stationary process for variance of the possible values of its parameter at a given instant of time, $K_{X}(0)=\sigma_{X}^{2}$. Then we can write:

$$
\sigma_{\mathrm{XB}}^{2}=\sigma_{\mathrm{X} 0}^{2} \cdot\left(\frac{1-\beta}{1+\beta}\right)^{10}
$$

For an arbitrary number of stages, we obtain the following system of equations:

$$
\left\{\begin{array}{l}
X_{1}=X_{0}+\beta \cdot X_{1} \\
X_{k}=(1-\beta) \cdot X_{k-1}+\beta \cdot X_{k}, \quad k=2,3, \ldots, n \\
X_{B}=(1-\beta) \cdot X_{n}
\end{array}\right.
$$

The system of equations that determine the correlation functions of material flows:

$$
\left\{\begin{array}{l}
K_{X 1}(\tau)=K_{X 0}(\tau)+\beta^{2} \cdot K_{X B 1}(\tau), \\
K_{X k}(\tau)=(1-\beta)^{2} \cdot K_{X B(k-1)}(\tau)+\beta^{2} \cdot K_{X B k}(\tau), k=2,3, \ldots, n, \\
K_{X B}(\tau)=(1-\beta)^{2} \cdot K_{X B n}(\tau)
\end{array}\right.
$$

The assumption is that $K_{X B i}(\tau)=K_{X i}(\tau)$, we obtain:

$$
\left\{\begin{array}{l}
K_{X 1}(\tau)=K_{X 0}(\tau)+\beta^{2} \cdot K_{X 1}(\tau), \\
K_{X k}(\tau)=(1-\beta)^{2} \cdot K_{X(k-1)}(\tau)+\beta^{2} \cdot K_{X k}(\tau), k=2,3, \ldots, n, \\
K_{X B}(\tau)=(1-\beta)^{2} \cdot K_{X n}(\tau)
\end{array}\right.
$$

Solving the system (8), we obtain:

$$
\mathrm{K}_{\mathrm{XB}}(\tau)=\mathrm{K}_{\mathrm{X} 0}(\tau) \cdot\left(\frac{1-\beta}{1+\beta}\right)^{\mathrm{n}}
$$

or:

$$
\sigma_{X B}^{2}=\sigma_{X 0}^{2} \cdot\left(\frac{1-\beta}{1+\beta}\right)^{n}
$$

When the numerical value of the recycling factor $\beta$ was substituted into formula (10), the dispersion ratio and the numerical value of the smoothing power were obtained for each cell of the drum mixer. For example, for a value of $\beta=$ 0.15 (corresponding to a drum filling factor of $24 \%$ ), the ratio of the variances on the first cell is $\sigma_{X 0}^{2} / \sigma_{X B}^{2}=0.74$, and on the fifth $\sigma_{X 0}^{2} / \sigma_{X B}^{2}=0.22$. Therefore, the dispersion of the outflow decreases significantly with the increasing number of recycles of material flows in the mixer. This indicates their influence on the smoothing ability, which is determined by the ratio $S=\sigma_{X 0}^{2} / \sigma_{X B}^{2}$, the numerical values of which are presented in Table 1 .

TABLE I. VALUES OF THE SMOOTHING CAPACITY OF THE DRUM MIXERWITH RECYCLE ON EACH BLADE

\begin{tabular}{|c|c|c|c|}
\hline $\begin{array}{l}\text { Number of } \\
\text { mixer blades }\end{array}$ & $\boldsymbol{\beta}$ & $\sigma_{X 0}^{2} / \sigma_{X B}^{2}$ & $\mathbf{S}$ \\
\hline 1 & 0.15 & 0.74 & 1.35 \\
\hline 2 & 0.15 & 0.54 & 1.83 \\
\hline 3 & 0.15 & 0.40 & 2.47 \\
\hline 4 & 0.15 & 0.29 & 3.35 \\
\hline 5 & 0.15 & 0.22 & 4.53 \\
\hline 6 & 0.15 & 0.16 & 6.13 \\
\hline 7 & 0.15 & 0.12 & 8.30 \\
\hline 8 & 0.15 & 0.08 & 11.23 \\
\hline 9 & 0.15 & 0.06 & 15.2 \\
\hline 10 & 0.15 & 0.04 & 20.57 \\
\hline
\end{tabular}

It can be seen from the table that the presence of reverse recirculations performed on each mixer blade significantly reduces the dispersion of the outflow stream[7,14]. At the same time, the smoothing capacity of the drum apparatus increases from 1.35 to 20.5 . 
Thus, the organization of directed movement of material flows (the presence of recirculation) [1,13], without taking into account the process of their averaging at the stages of the mixer, allows one several times to reduce the dispersion of the components of the mixture at the outlet from it and, as a result, to increase its homogeneity.

Then we analyze the second scheme, shown in Fig. 3, in which the material flows are recirculated and then superimposed through one blade (cell) of the drum mixer. The material balance in this case is described by the following equations:

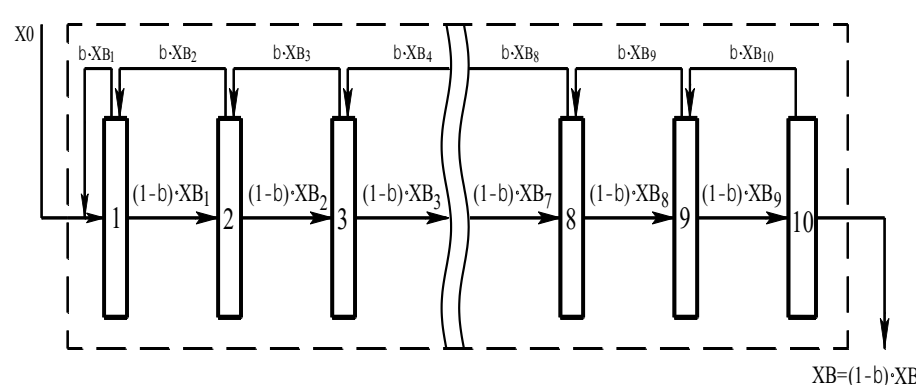

Fig. 2 The scheme of motion of material flows in the staggered arrangement of $\Gamma$-shaped blades in a drum mixer

$$
\left\{\begin{array}{l}
X_{1}=X 0+\beta \cdot X_{1} \\
X_{2}=(1-\beta) \cdot X_{1} \\
X_{3}=(1-\beta) \cdot X_{2}+\beta \cdot X_{3} \\
X_{4}=(1-\beta) \cdot X_{3} \\
X_{5}=(1-\beta) \cdot X_{4}+\beta \cdot X_{5} \\
X_{6}=(1-\beta) \cdot X_{5} \\
X_{7}=(1-\beta) \cdot X_{6}+\beta \cdot X_{7} \\
X_{8}=(1-\beta) \cdot X_{7} \\
X_{9}=(1-\beta) \cdot X_{8}+\beta \cdot X_{9} \\
X_{10}=(1-\beta) \cdot X_{9} \\
X B=(1-\beta) \cdot X_{10}
\end{array}\right.
$$

We write down the system of equations that determine the correlation functions of the flows, taking into account the fact that $\mathrm{K}_{\mathrm{XBi}}(\tau)=\mathrm{K}_{\mathrm{Xi}}(\tau)$ :

$$
\left\{\begin{array}{l}
\mathrm{K}_{\mathrm{X} 1}(\tau)=\mathrm{K}_{\mathrm{X} 0}(\tau)+\beta^{2} \cdot \mathrm{K}_{\mathrm{X} 1}(\tau), \\
\mathrm{K}_{\mathrm{X} 2}(\tau)=(1-\beta)^{2} \cdot \mathrm{K}_{\mathrm{X} 1}(\tau) \\
\mathrm{K}_{\mathrm{X} 3}(\tau)=(1-\beta)^{2} \cdot \mathrm{K}_{\mathrm{X} 2}(\tau)+\beta^{2} \cdot \mathrm{K}_{\mathrm{XB} 3}(\tau), \\
\mathrm{K}_{\mathrm{X} 4}(\tau)=(1-\beta)^{2} \cdot \mathrm{K}_{\mathrm{X} 3}(\tau) \\
\mathrm{K}_{\mathrm{X} 5}(\tau)=(1-\beta)^{2} \cdot \mathrm{K}_{\mathrm{X} 4}(\tau)+\beta^{2} \cdot \mathrm{K}_{\mathrm{XB}}(\tau), \\
\mathrm{K}_{\mathrm{X} 6}(\tau)=(1-\beta)^{2} \cdot \mathrm{K}_{\mathrm{X} 5}(\tau) \\
\mathrm{K}_{\mathrm{X} 7}(\tau)=(1-\beta)^{2} \cdot \mathrm{K}_{\mathrm{X} 6}(\tau)+\beta^{2} \cdot \mathrm{K}_{\mathrm{XB} 7}(\tau), \\
\mathrm{K}_{\mathrm{X} 8}(\tau)=(1-\beta)^{2} \cdot \mathrm{K}_{\mathrm{X} 7}(\tau) \\
\mathrm{K}_{\mathrm{X} 9}(\tau)=(1-\beta)^{2} \cdot \mathrm{K}_{\mathrm{X} 8}(\tau)+\beta^{2} \cdot \mathrm{K}_{\mathrm{XB} 9}(\tau), \\
\mathrm{K}_{\mathrm{X} 10}(\tau)=(1-\beta)^{2} \cdot \mathrm{K}_{\mathrm{X} 9}(\tau) \\
\mathrm{K}_{\mathrm{XB}}(\tau)=(1-\beta)^{2} \cdot \mathrm{K}_{\mathrm{X} 10}(\tau)
\end{array}\right.
$$

The result of the solution of system (12) is the following expression:

$$
\mathrm{K}_{\mathrm{XB}}(\tau)=\mathrm{K}_{\mathrm{X} 0}(\tau) \cdot\left(\frac{1-\beta}{1+\beta}\right)^{5}
$$

or considering that $\mathrm{K}_{\mathrm{X}}(0)=\sigma_{\mathrm{X}}^{2}$ :

$$
\sigma_{\mathrm{XB}}^{2}=\sigma_{\mathrm{X} 0}^{2} \cdot\left(\frac{1-\beta}{1+\beta}\right)^{5} \text {. }
$$

We write the following system of equations for an arbitrary number of stages:

$$
\left\{\begin{array}{l}
X_{1}=X_{0}+\beta \cdot X_{1} \\
X_{k}=(1-\beta) \cdot X_{k-1}, \quad k=2,4,6, \ldots, n \\
X_{k}=(1-\beta) \cdot X_{k-1}+\beta \cdot X_{k}, \quad k=3,5,7, \ldots n-1, \\
X_{B}=(1-\beta) \cdot X_{n}
\end{array}\right.
$$

The system of equations that determine the correlation functions of flows under the $\mathrm{K}_{\mathrm{XBi}}(\tau)=\mathrm{K}_{\mathrm{Xi}}(\tau)$ will have the form:

$$
\left\{\begin{array}{l}
\mathrm{K}_{\mathrm{X} 1}(\tau)=\mathrm{K}_{\mathrm{X} 0}(\tau)+\beta^{2} \cdot \mathrm{K}_{\mathrm{X} 1}(\tau), \\
\mathrm{K}_{\mathrm{Xk}}(\tau)=(1-\beta)^{2} \cdot \mathrm{K}_{\mathrm{X}(\mathrm{k}-1)}(\tau), \quad \mathrm{k}=2,4,6, \ldots, \mathrm{n}, \\
\mathrm{K}_{\mathrm{Xk}}(\tau)=(1-\beta)^{2} \cdot \mathrm{K}_{\mathrm{X}(\mathrm{k}-1)}(\tau)+\beta^{2} \cdot \mathrm{K}_{\mathrm{Xk}}(\tau), \quad \mathrm{k}=3,5,7 \ldots, \mathrm{n}-1, \\
\mathrm{~K}_{\mathrm{XB}}(\tau)=(1-\beta)^{2} \cdot \mathrm{K}_{\mathrm{Xn}}(\tau)
\end{array}\right.
$$

Solving the system (16), we obtain (for an even number of blades):

$$
\mathrm{K}_{\mathrm{XB}}(\tau)=\mathrm{K}_{\mathrm{X0}}(\tau) \cdot\left(\frac{1-\beta}{1+\beta}\right)^{\frac{\mathrm{n}}{2}}
$$

or:

$$
\sigma_{\mathrm{XB}}^{2}=\sigma_{\mathrm{X} 0}^{2} \cdot\left(\frac{1-\beta}{1+\beta}\right)^{\frac{n}{2}} \text {. }
$$

For an odd number of blades:

$$
\mathrm{K}_{\mathrm{XB}}(\tau)=\mathrm{K}_{\mathrm{X} 0}(\tau) \cdot\left(\frac{1-\beta}{1+\beta}\right)^{\frac{\mathrm{n}-1}{2}}
$$

or:

$$
\sigma_{X B}^{2}=\sigma_{X 0}^{2} \cdot\left(\frac{1-\beta}{1+\beta}\right)^{\frac{n-1}{2}} \text {. }
$$

Substituting into the equation (20), the numerical value of the recirculation coefficient equal to 0.17 (corresponding to a drum filling factor of $24 \%$ ), we obtain that the dispersion ratio, for example, in the first cell is $\sigma_{X 0}^{2} / \sigma_{X B}^{2}=1$, and on the seventh $\sigma_{X 0}^{2} / \sigma_{X B}^{2}=0,357$, which indicates a decrease in the dispersion of the outflow with an increase in the number of recycles in the apparatus. This confirms the fact of their effect 
on the smoothing ability of S, the numerical values of which are presented in Table 2.

It can be seen from the table that the dispersion values of the outflow and the smoothing capacity of the apparatus repeat their numerical values on each odd blade (cell). This is confirmed by the fact that recirculation of the mixture occurs through one blade, namely at each even cell. In this case, the smoothing capacity of the drum machine increases from the beginning of the mixer to its end in the range from 1 to 5.565 .

TABLE II. VALUES OF THE SMOOTHING CAPACITY OF THE DRUM MIXER WITH RECYCLING THROUGH ONE BLADE

\begin{tabular}{|c|c|c|c|}
\hline $\begin{array}{l}\text { Number of } \\
\text { mixer blades }\end{array}$ & $\boldsymbol{\beta}$ & $\sigma_{X 0}^{2} / \sigma_{X B}^{2}$ & $\mathbf{S}$ \\
\hline 1 & 0.17 & 1 & 1 \\
\hline 2 & 0.17 & 0.709 & 1.409 \\
\hline 3 & 0.17 & 0.709 & 1.409 \\
\hline 4 & 0.17 & 0.503 & 1.987 \\
\hline 5 & 0.17 & 0.503 & 1.987 \\
\hline 6 & 0.17 & 0.357 & 2.8 \\
\hline 7 & 0.17 & 0.357 & 2.8 \\
\hline 8 & 0.17 & 0.253 & 3.948 \\
\hline 9 & 0.17 & 0.253 & 3.948 \\
\hline 10 & 0.17 & 0.179 & 5.565 \\
\hline
\end{tabular}

Analysis of the results of the calculation of the two circuits shows that the best smoothing ability is possessed by a mixer operating according to the first scheme (in which the mixture is displaced longitudinally in the apparatus, undergoing recirculation in each of its cells). The smoothing ability of the drum mixer operating under this scheme is 3.68 times higher $(S=20.5)$ than the second $(S=5.565)$, which recirculates material flows through one blade. Therefore, to obtain more homogeneous it is expedient to carry out the process of mixing in a drum mixer by the installation of $\Gamma$-shaped blades in a spiral manner (with recirculation on each blade).

\section{CONCLUSION}

1. Theoretical analysis of a continuous drum mixer with various schemes of the organization of the flow of material flows in it, based on the theory of random processes.

2. As a result of the correlation analysis of the two schemes of material movement, it is established that the organization of external and internal recirculation circuits in the apparatus has a rather strong effect on reducing the fluctuations of the input signal. This allows one several times to reduce the dispersion of the components of the mixture after they pass through the drum mixer and, as a result, increase its homogeneity.

\section{References}

[1] T.Akiyama, H.Kurimoto, "Compressible Gas Model of Vibrated Particale Beds”, Chem. Eng. Scien, 1988, vol.43, pp. 2645-2653.

[2] R.Akkerman, D.Van Der Meer, D.P.Van Donk, "Make to stock and mix to order: choosing intermediate products in the food-processing industry", International Journal of Production Research, 2010, vol. 48, no. 12 , pp. 3475-3492.

[3] D.Bendall, "Mixers and food processors", Food Management, 2003, vol. 38 , no. 2 , p. 74

[4] F. Berruti, A.G. Liden, D.S.Scott, "Measuring and Modeling Residence Time Distribution of Low Density Solid in a Fluidized Bed Reator of Sand Particles”, Chem. Eng. Scien.,1988, vol.43, pp.739-748.

[5] D.M. Borodulin, D. VOL. Sukhorukov, "Mixtures for vertebroplasty: the flowability of their components and a new production technology using a centrifugal mixer", Foods and Raw Materials, 2014, no. 1, pp. 67-71.

[6] D. Brone, A. Alexander, F. Muzzio, "Quantitative characterization of mixing of dry powders in V-blenders", AICHE, 1998, no. 44 (2), pp. 271-278.

[7] J. L. Chen, "A theoretical model for particle segregation in a fluidized bed due to size difference", Chem. Eng. Commun, 1981, no. 9, pp. 303 320 .

[8] C. Harwood, K. Walanski, E. Luebcke, "The Performance of Continuous mixers for dry powders", Powder Technology, 1975, no. 11, pp. 289 296.

[9] V.N. Ivanets, D.M. Borodulin, A.B. Shushpannikov, D. V. Sukhorukov "Intensification of bulk material mixing in new designs of drum, vibratory and centrifugal mixers", Foods and Raw Materials, 2015, no. $3(1)$, p. $62-69$

[10] O.Krasulya, S.Shestakov, V. Bogush, I. Potoroko, P. Cherepanov, B.Krasulya, "Applications of sonochemistry in russian food processing industry”, Ultrasonics Sonochemistry, 2014, vol. 21, no. 6, pp. 2112 2116.

[11] V. Kuzmichev, V. Verstov, "Gravity mixers with vibration activator in construction engineering”, Architecture and engineering, 2017, no. 2 (4), pp. 26-33.

[12] A.M. Popov, "Systemic regularities in the study and design of technological complexes for the production of instant beverages", Foods and Raw Materials, 2014, vol. 2, no. 2, pp. 156-160.

[13] G. Ratushnyak, V. Djedjula, "Modelling of processes of heat exchange at vibrating influence in multicomponent mixtures of bioreactors", Scientific works of vinnytsia national technical university, 2008, no. 1, p. 10

[14] S. Tallon, C.E. Davies "In-situ monitoring of axial particle mixing in a rotating drum using bulk density measurements", Powder Technology, 2008 , vol. 186 , no. 1 , pp. 22-30.

[15] D.R.Van Puyvelde, "Simulating the mixing and segregation of solids in the transverse section of a rotating kiln", Powder Technology, 2006, vol. 164 , no. 1 , pp. 1-12. 